\title{
Established and potential echocardiographic markers of embolism and their therapeutic implications in patients with ischemic stroke
}

\author{
Paulina E. Gąsiorek ${ }^{1}$, Maciej Banach ${ }^{2}$, Marek Maciejewski ${ }^{3}$, Andrzej Głąbiński ${ }^{1}$,
} Aleksandra Paduszyńska ${ }^{2}$, Jacek Rysz ${ }^{4}$, Agata Bielecka-Dąbrowa ${ }^{2}$

${ }^{1}$ Department of Neurology and Ischemic Strokes, Medical University of Lodz, Poland ${ }^{2}$ Department of Hypertension, Chair of Nephrology and Hypertension, Medical University of Lodz, Poland

${ }^{3}$ Department of Cardiology and Congenital Diseases of Adults, ICMP, Lodz, Poland

${ }^{4}$ Department of Nephrology, Hypertension and Family Medicine, Chair of Nephrology and Hypertension, Medical University of Lodz, Poland

\begin{abstract}
Cardiogenic strokes comprised $11 \%$ of all strokes and $25 \%$ of ischemic strokes. An accurate identification of the cause of stroke is necessary in order to prepare an adequate preventive strategy. In this review the confirmed and potential causes of embolic strokes are presented, which can be detected in echocardiography in the context of present treatment guidelines and gaps in evidence. There remains a need for further studies assessing the meaning of potential cardiac sources of embolism and establishment of rules for optimal medical prevention (antiplatelet therapy [APT] vs. oral anticoagulation [OAC]) and interventional procedures to reduce the incidence of ischemic strokes. Currently available data does not provide definitive evidence on the comparative benefits of OAC vs. APT in patients with cryptogenic stroke or embolic stroke of undetermined source. There is a lack of antithrombotic treatment scheme in the time between stroke and the completed diagnosis of potential sources of thromboembolism. (Cardiol J 2019; 26, 5: 438-450)
\end{abstract}

Key words: cardioembolic stroke, anticoagulant therapy, cardiac sources of embolism, ischemic stroke, cryptogenic stroke

\section{Introduction}

Approximately 1.1 million inhabitants of Europe suffera stroke each year, and ischemic stroke accounted for approximately $80 \%$ of cases $[1,2]$. Although global stroke incidence is declining, rates observed in young adults are on the rise, thus suggesting a need for strategies to improve prevention [2]. In addition, because of an ageing population, the absolute number of strokes is expected to dramatically increase in coming years: by 2025, 1.5 million European people will suffer a stroke each year. Stroke is associated with increased long-term mor- tality. Beyond vital prognosis, stroke patients are also at increased risk of poor outcome within the first year of the event including re-hospitalization (33\%), recurrent event (7\% to 13\%), dementia (7\% to $23 \%$ ), mild cognitive disorder (35\% to $47 \%$ ), depression (30\% to $50 \%$ ), and fatigue (35\% to $92 \%$ ), which all contribute to, and affect health related quality of life. Ischemic stroke is a heterogeneous disease with different mechanisms and etiologies and their specific treatments. An accurate identification of the cause is essential in order to prepare an adequate preventive strategy. A substantial proportion of stroke risk remains unexplained [3].

Address for correspondence: Paulina Gąsiorek, MD, PhD, Department of Neurology and Ischemic Strokes, Medical University of Lodz, ul. Żeromskiego 113, 90-549 Łódź, Poland, e-mail: paulinka.com@gmail.com 
Using the Trial of Org 10172 in Acute Stroke Treatment (TOAST) criteria, ischemic strokes may be further subdivided into following types:

1. Thrombosis or embolism associated with large vessel atherosclerosis;

2. Embolism of cardiac origin (cardioembolic stroke);

3. Small blood vessel occlusion (lacunar stroke);

4. Other determined cause;

5. Undetermined (cryptogenic) cause (no cause identified, more than one cause, or incomplete investigation) [4].

The incidence of each cause is variable and depends on patient age, sex, race, geographic location, risk factors, clinical history, physical findings, and results of various tests. Embolism of cardiac origin accounts for around $15-30 \%$ of ischemic strokes. It is worth noting that stroke from a cardiac source carries a poorer outcome compared with other sources, having a 50\% mortality at 3 years. The diagnosis of a cardioembolic source of stroke is frequently uncertain and relies on the identification of a potential cardiac source of embolism in the absence of significant autochthone cerebrovascular occlusive disease [5]. While a stuttering course has usually been attributed to atherothrombotic stroke, cardioembolic strokes can have a progressive course in at least one-fifth of cases given that emboli can recanalize, move and fragment after initial impaction. Rapid regression of symptoms (the spectacular shrinking syndrome) reflecting early recanalization has also been related to cardioembolic stroke [6].

In patients who are at risk for or have already had potentially embolic strokes, the primary role of echocardiography is to establish the existence of the source of embolism, determine the likelihood that such a source is a plausible cause of stroke or systemic embolism, and guide therapy in an individual patient. Ultimately stroke is classified as cryptogenic or undetermined when no cause is identified after a thorough study [7]. Embolic stroke of undetermined source (ESUS) is onefourth among cerebral infarction, but most of them could be ascribed to embolic stroke. This review presents the confirmed and potential causes of embolic strokes which can be detected in echocardiography in the context of present treatment guidelines and gaps in evidence. There is still a need for further studies assessing the meaning of potential cardiac sources of embolism and establishment of the rules of the optimal medical prevention (antiplatelet therapy [APT] vs. oral anticoagulation $[\mathrm{OAC}]$ ) and interventional procedures to reduce the incidence of ischemic strokes.

\section{Cryptogenic stroke and ESUS}

The term cryptogenic stroke has been extensively used in the literature to describe ischemic strokes of undetermined etiology. However, this does not take into account the extent and quality of the investigation performed or classification system used [8]. In the TOAST classification system, stroke of undetermined cause may refer to a stroke with incomplete workup, more than one potential cause, or indeed no determined etiology after investigations are complete [9]. In 2014, the term ESUS, was coined by the cryptogenic stroke /ESUS international working group, ESUS refers to non-lacunar infarct (subcortical infarct $\leq 1.5 \mathrm{~cm}$ on computed tomography [CT] or $\leq 2.0 \mathrm{~cm}$ on magnetic resonance imaging $[\mathrm{MRI}])$ in the absence the following: extracranial or intracranial atherosclerosis causing $>50 \%$ luminal stenosis in the artery supplying the ischemic region, major cardioembolic sources permanent or paroxysmal atrial fibrillation (AF), sustained atrial flutter, intracardiac thrombus, prosthetic cardiac valve, atrial myxoma or other cardiac tumors, mitral stenosis (MS), myocardial infarction (MI) within the past 4 weeks, left ventricular (LV) ejection fraction $(\mathrm{LVEF})<30 \%$, valvular vegetation's or infective endocarditis (IE), and no other specific cause of stroke (e.g., dissection, arteritis, migraine/ vasospasm, drug misuse) [7]. Extensive evaluation including transesophageal echocardiography (TEE) and cardiac monitoring over an extended period of time could identify the etiology of ESUS patients. Although an antiplatelet drug is recommended in ESUS is the current guideline, clinical trials are ongoing to determine the efficacy of non-vitamin K antagonist oral anticoagulant in ESUS patients [9].

\section{The established and potential cardiac reasons of ischemic stroke}

Echocardiography (both, transthoracic echocardiography [TTE] and TEE) is a widely used and versatile technique that can provide comprehensive information of thromboembolic risk in patients with stroke $[8,9]$. In many conditions more than one embolic source may be present (coexistence of embolic sources) or one cardioembolic condition may lead to another (interdependent of embolic sources). For instance, MS is associated with spon- 
taneous echocardiographic contrast, AF, clot, and even endocarditis. Conditions that are known to lead to systemic embolization are subdivided into high-risk and low-risk groups on the basis of their embolic potential and are presented in Tables 1 and $2[6,8]$.

\section{The established cardiac sources of ischemic stroke}

\section{Atrial fibrillation}

Atrial fibrillation is the most common cardiac arrhythmias, and a major cause of morbidity and mortality due to cardioembolic stroke. Prevalence of $\mathrm{AF}$ increases with age, up to $15 \%$ in octogenerians, and continues to grow rapidly due to the increasing proportion of aging in the population. Moreover, the elderly population of today has a higher prevalence of predisposing conditions for $\mathrm{AF}$, such as diabetes, heart failure, hypertension, and coronary heart disease [10]. Newly detected $\mathrm{AF}$ is identified in $10 \%$ of the patients who experience a stroke or a transient ischemic attack (TIA), and an additional $11 \%$ cases of $\mathrm{AF}$ are newly detected when patients undergo 30 days of continuous electrocardiographic monitoring [11]. The left atrial appendage (LAA) is the major site of thrombus formation in non-valvular AF. TEE is considered to be the gold-standard technique to detect LAA thrombi, with values of sensitivity and specificity approaching 99\% [11].

In the Stroke Prevention in Atrial Fibrillation (SPAF) III and other trials, LAA thrombi, dense spontaneous echo contrast, LAA peak flow velocities $20 \mathrm{~cm} / \mathrm{s}$, and complex aortic plaques were independently associated with increased thromboembolic risk in $\mathrm{AF}$ patients [12].

Atrial fibrillation detection is important in stroke prevention, because anticoagulation is known to decrease stroke risk in the presence of this arhythmia. A meta-analysis of 50 studies involving more than 10,000 patients with a recent stroke found that $7.7 \%$ had $\mathrm{AF}$ on their admitting electrocardiogram. Within 3 weeks during and after hospitalization, another $16.9 \%$ were diagnosed. A total of $23.7 \%$ of these stroke patients had silent $\mathrm{AF}$; that is AF diagnosed after hospital admission [13]. Olsen et al. [14] aimed to evaluate whether speckle tracking echocardiography improves risk stratification for AF. After 5.5 years patients who presented a composite of new-onset $\mathrm{AF}$ and ischemic stroke had significantly reduced systolic function by LVEF and global longitudinal strain, however, only global
Table 1. The conditions with a high embolic potential.

\begin{tabular}{l} 
The sources of intracardiac thrombi \\
Atrial arrhythmias: \\
- Valvular atrial fibrillation \\
- Non-valvular atrial fibrillation \\
- Atrial flutter \\
Akinetic segments and diffuse \\
ventricular hypokinesia: \\
- Recent myocardial infarction \\
- Ventricular aneurysm \\
- Non-ischemic cardiomyopathies \\
Prosthetic valves and devices \\
Intracardiac vegetations \\
Myxoma \\
Papillary fibroelastoma \\
Other tumors \\
Intracardiac vegetations \\
Infective endocarditis \\
Marantic endocarditis \\
Aortic atheroma \\
Thromboembolism \\
Cholesterol crystal emboli \\
\hline
\end{tabular}

Table 2. The conditions with a low embolic potential.

Septal defects and anomalies
Patent foramen ovale
Atrial septal defect
Atrial septal aneurysm
Intracardiac calcifications
Mitral annular calcifications, mitral stenosis (mainly
in the course of rheumatic heart disease)
Calcific aortic stenosis
Substenotic atherosclerotic plaques
Potential precursors of intracardiac thrombi
Mitral valve prolapse
Pouches of atrial septum
Enlargement of left atrium
Structures in dextral atrium like Eustachian valve
or Chiari's network

longitudinal strain remained a significantly independent predictor (hazard ratio [HR] 1.12, 95\% confidence interval [CI] 1.00-1.25, $\mathrm{p}=0.042$, per $1 \%$ decrease) after multivariable adjustment for baseline predictors (age, sex, diabetes, hypertension, diastolic dysfunction, and LVEF) [14]. 


\section{Myocardial infraction} and cardiomyopathies

Myocardial infarction and dilated cardiomyopathy (DCM) are most frequently associated with LV thrombus formation attributable to stasis caused by regional or global myocardial dysfunction. From $1 \%$ to $2.5 \%$ of patients with acute MI suffer a stroke within 4 weeks, one-half in the first 5 days. LV thrombus was more likely in patients with $\mathrm{EF}$ of $<40 \%$, anterior wall MI and LV aneurysm [11]. Embolic events are estimated to occur in $4 \%$ of patients with DCM who have a LVEF $\leq 35 \%$. Further, the incidence of LV thrombus in patients with DCM and sinus rhythm is $13 \%$, and DCM may also have a high incidence of clinically asymptomatic silent cerebral infarction [15]. Isolated LV noncompaction (LVNC) is a genetic cardiomyopathy characterized by prominent ventricular trabeculations and deep intertrabecular recesses, or sinusoids, in communication with the LV cavity. The clinical sequelae of these deformities are the syndrome of heart failure and the risk for arrhythmias and stroke. The diagnostic studies for evaluation of LVNC are echocardiography, cardiac magnetic resonance (CMR) and cardiac CT. The Stöllberger et al. [16] revealed that stroke/ /embolism in LVNC is not always cardioembolic, but may also have an atherosclerotic cause. The $\mathrm{CHADS}_{2}$ score may be useful for clinical decisionmaking about OAC for the prevention of stroke/ /embolism in LVNC patients [17].

\section{Rheumatic heart disease}

Rheumatic heart disease is a common cause of stroke in developing countries. Among patients with rheumatic heart disease, MS is the valve lesion most strongly associated with stroke and systemic embolism [18, 19]. Studies from the middle part of the last century found an annual incidence of systemic embolism among patients with rheumatic mitral valve disease of $1.5 \%$ to $4.7 \%$ [20]. Reports on the association of embolic stroke with mitral valve prolapse have been inconsistent [19-21]. A population-based study of patients from Olmsted County, Minnesota, found an increased risk ratio (RR) of stroke or TIA among patients with mitral valve prolapse who were initially in sinus rhythm (RR 2.2; $95 \%$ CI 1.5-3.2) [21]. The presence of left atrium enlargement and $\mathrm{AF}$ increase the incidence of silent brain infarction in patients with MS, whereas the presence of moderate to severe mitral regurgitation decreases the incidence [21].

\section{Endocarditis}

Stroke often complicates IE, affecting 16-25\% of patients with IE [21].

Most diagnoses of stroke and IE are made close together in time, but a period of heightened stroke risk becomes apparent several months before the diagnosis of IE and lasts for several months afterward [22, 23]. In the study of Merkler et al. [24] among 17,926 patients with IE, 2,275 strokes occurred within the 12 -month period surrounding the diagnosis of IE.

The risk of stroke was highest in the month after diagnosis of IE (1,640 vs. 17 strokes in the corresponding month 2 years prior) [24]. A transthoracic echocardiography must be performed in every case of suspected IE. Vegetations (infected tissue mass) appear as irregular echogenic masses usually attached to the atrial side of the atrioventricular valves or the ventricular side of the semilunar valves along the line of leaflet coaptation. The vegetations are seen to move independently to the underlying valve motion [15]. IE requires administration of systemic antibiotics and, if indicated, surgery. Non-bacterial thrombotic endocarditis (NBTE), ormarantic endocarditis, is composed of small, sterileplatelet - fibrin vegetations on the cardiac valves. There is little inflammatory reaction at the foot of the growth so the thrombi detach easily, causing multiorgan infarcts. The pathogenesis of NBTE is not completely understood. It is particularly associated with adenocarcinomas of the gastrointestinal tract and lungs and mechanisms are considered to be similar to the ones underlying cancer hypercoagulability [21, 23]. A clinical triad of a known disease process associated with NBTE, heart murmur and signs of multiple systemic emboli should alert the physician to NBTE. NBTE could be treated with tumor-suppressive, antiretroviral, or immunosuppressive therapy with systemic anticoagulation, preferably heparin-based however treatment options are limited because of Disseminated Intravascular Coagulation which is often present.

Fibrous and fibrinous lesions of the heart valves and endocardium traditionally occur in patients with systemic lupus erythematosus (Libman-Sacks endocarditis), antiphospholipid antibody syndrome. Fibrin-platelet aggregates may attach to these fibrous and fibrinous lesions [23, 24].

\section{Cardiac tumors \\ Primary tumors of the heart are rare with the incidence of $0.02-0.05 \%$ [25].}


The majority of them are myxomas, accounting for only $0.02 \%$ of primary tumors, and $75 \%$ of them are benign. Embolic manifestations occur in $20-45 \%$ of patients with a cardiac myxoma, sometimes as the onset symptom [26]. From $75 \%$ to $80 \%$ of myxomas are located in the left atrium. Myxomas arising from the right ventricle are extremely rare. Most cases (90\%) of atrial myxoma are sporadic with no known cause. In the remaining (10\%), a familial pattern occurs having an autosomal dominant pattern known as Carney complex which is characterized by multiple tumors, including atrial and extracardiac myxomas, cutaneous spotty pigmentation, non-myxomatous extracardiac tumors, schwannomas and various endocrine tumors, through a causative mutation of the PRKAR $1 \alpha$ gene located on the long arm of chromosome 17 [25]. Cardiac myxomas produce interleukin (IL)-6, leading to constitutional manifestations such as recurrent fever, anemia, arthralgia, and weight loss which disappear after removal of the tumor [26, 27]. Myxomas are frequently found particularly between the third and sixth decades of life. There are 2 types of cardiac myxomas: friable polypoid type and smooth-surface rounded type. The polypoidal type tends to result in embolism because of its friable consistency and intracavitary location [28]. Furthermore, the LV has a higher risk of embolization resulting from high mobility and pressure in it $[29,30]$. The second most common benign primary tumor of the heart is papillary fibroelastoma. Its prevalence is $0.002 \%$ to $0.28 \%$ among the general population. The average age at diagnosis is 56 , with a primarily male preponderance (58\%). These kinds of tumors are often found on cardiac valvular surfaces. The clinical presentation of fibroelastoma varies widely, ranging from clinically asymptomatic to severe thromboembolic events. It is important to differentiate fibroelastomas from cardiac myxomas and thrombi because of differences in their medical treatment. A clear surgical margin is necessary for excision of a myxoma due to its high rate of recurrence. On the other hand, fibroelastomas rarely recur after resection, thus it is recommended to preserve valvular function by shaving off the tumor [25]. Diagnosis at present is established most appropriately with two dimensional echocardiography. CMR can help out in demarcating tumor size, attachment and its mobility and this information may be further used during surgical resection. Positron emission tomography scan has been useful in identifying cardiac involvement in patients with metastatic tumors and atrial myxoma [29]. Although, since tumor fragments may embolise, early anticoagulation may not be protective in reducing disability and mortality [30]. Prompt excision using cardiopulmonary bypass, first carried out by Crafoord in 1954, has been established as the only acceptable mode of treatment for these tumors. The results of surgical resection are generally very good, with most series reporting an operative death rate of $<5 \%$.

\section{Potential sources of thromboembolism}

\section{Patent foramen ovale}

and atrial septal defect

Patent foramen ovale (PFO) is the most common congenital cardiac abnormality present in approximately $25 \%$ of the population and is responsible for up to $95 \%$ of right-to-left shunts (Fig. 1). $\mathrm{PFO}$ is a communication across the interatrial septum between a nonadherent septum primum and septum secundum and is considered to be a risk factor for serious clinical syndromes including paradoxical systemic embolism such as embolic strokes, MI, decompression sickness in divers, and complications of pulmonary embolism [27]. PFO prevalence among patients with cryptogenic stroke are typically high with median prevalence of approximately $40 \%$ and more commonly present in young people. Saline contrast injection or agitated saline mixed with air (also referred to as a "bubble study") during TTE or TEE can detect a PFO if microbubbles which are seen within the left atrial chamber within three cardiac cycles after right atrial opacification [8]. In situ thrombus formation and propensity for cardiac arrhythmias in patients with PFO have been suggested stroke mechanisms [1]. No association has been documented between shunt amount and stroke recurrence. Until now, whether PFO is a risk factor for stroke has been unsettled. In the SISIFO study - a multicenter, prospective, single-wave, cross-sectional survey conducted on 1,130 consecutive patients with acute ischemic stroke admitted to selected clinical centres underline PFO alone must not be considered a significant independent predictor for stroke; so the presence of PFO alone doesn't permit rushed causal correlations or 'therapeutic aggressiveness' [31]. By far, paradoxical embolization is the most commonly proposed mechanism for stroke in PFO patients. Therefore, finding a proximal source such as a deep venous thrombus is warranted, otherwise the association is rendered theoretical. Besides PFO closure did not prevent stroke. Clinical conditions such as deep vein thrombosis, a prolonged immobility/postopera- 


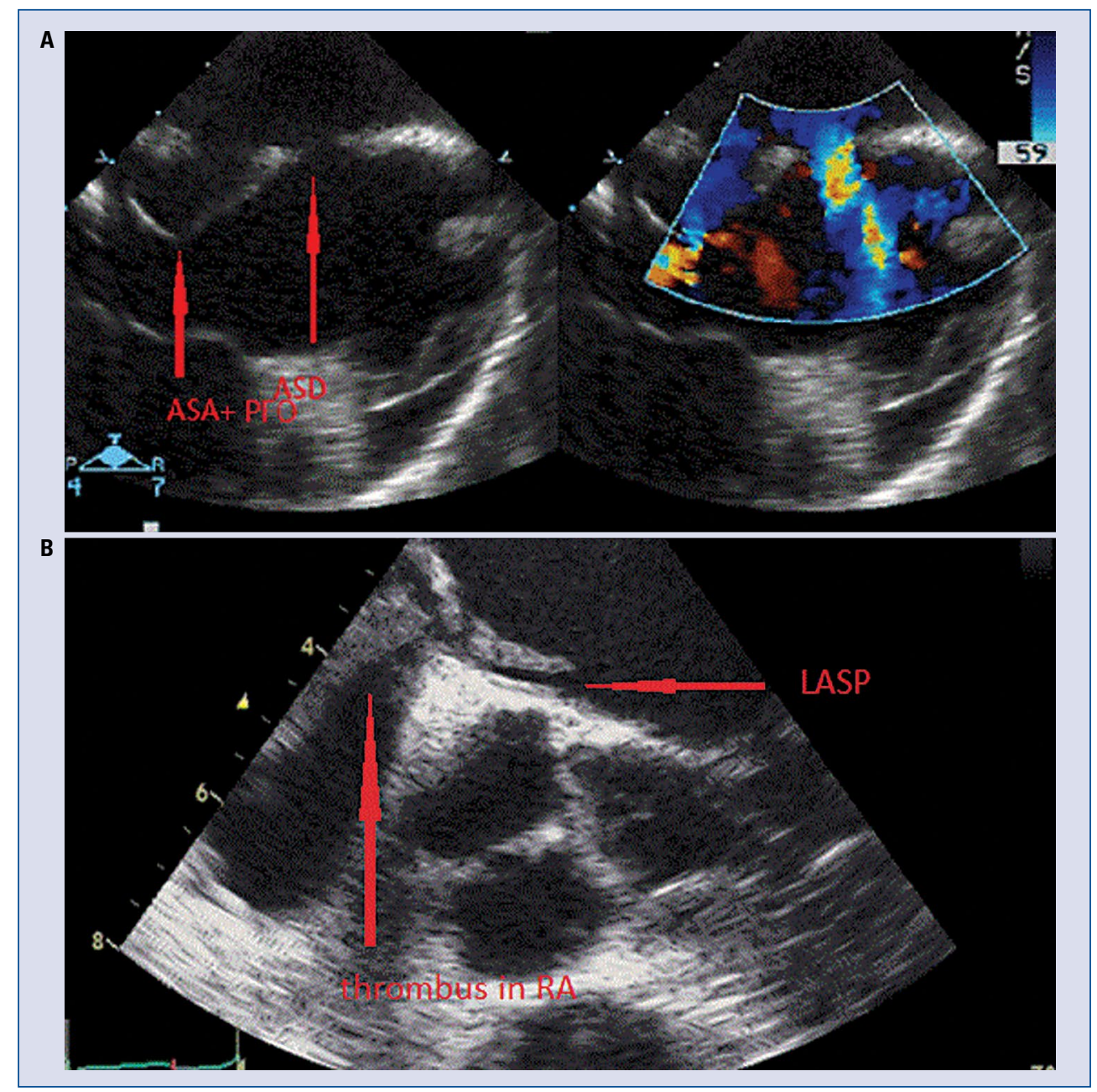

Figure 1. A. Transesophageal echocardiography (TEE), atrial septal aneurysm (ASA), patent foramen ovale (PFO) and atrial septal defect (ASD) in patient after ischemic stroke; B. TEE. The left atrial septal pouch (LASP) and mobile thrombus in right atrium (RA).

tive period, and the Valsalva maneuver were also not associated with embolism recurrence. Taken together, a mechanism other than paradoxical embolism may contribute to the development of embolic stroke in patients with PFO [32-34]. The probability of having a $\mathrm{PFO}$ as a possible cause of stroke in cryptogenic stroke patients may vary according to patient characteristics [35, 36]. Several factors possibly associated with increased risk of stroke recurrence in patients with PFO include a right-to-left shunt detectable in resting conditions, amount of right-to-left shunt under Valsalva, and a combination of PFO with either atrial septal aneurysm (ASA) or increased interatrial septal mobility (Fig. 1) [37-39]. Several clinical and brain imaging features, including the 10-point Risk of Paradoxical Embolism (RoPE) score, have been suggested to determine a high-risk PFO (Table 3) [39].

\section{Substenotic atherosclerotic plaques}

Substenotic atherosclerotic plaques can possibly cause ischemic stroke by plaque rupture and artery-to-artery embolization. In particular, complicated atherosclerotic plaques with evidence of intraplaque hemorrhage on imaging have been suggested to be a potential mechanism in ESUS [7].

There are more potential causes of cardiac strokes, which are not entirely examined as:

- pouches of atrial septum;

- structures in dextral atrium like Eustachian valve $(\mathrm{EV})$ or Chiari's network $(\mathrm{CN})$;

- enlargement of left atrium;

- ASA.

\section{Pouches of left atrial septum}

The left atrial septal pouch (LASP) was described in 2010 by Krishnan and Salazar as 
Table 3. Ten-point risk of paradoxical embolism (RopE) score to determine a high-risk patent foramen ovale (PFO).

\begin{tabular}{|c|c|c|}
\hline \multicolumn{2}{|c|}{ Characteristic } & Points \\
\hline \multicolumn{2}{|c|}{ No history of hypertension } & +1 \\
\hline \multicolumn{2}{|c|}{ No history of diabetes } & +1 \\
\hline \multicolumn{2}{|c|}{ No history of stroke or TIA } & +1 \\
\hline \multicolumn{2}{|c|}{ Nonsmoker } & +1 \\
\hline \multicolumn{2}{|c|}{ Cortical infarct on imaging } & +1 \\
\hline \multicolumn{3}{|c|}{ Age [years]: } \\
\hline \multicolumn{2}{|l|}{$18-29$} & +5 \\
\hline \multicolumn{2}{|l|}{$30-39$} & +4 \\
\hline \multicolumn{2}{|l|}{$40-49$} & +3 \\
\hline \multicolumn{2}{|l|}{$50-59$} & +2 \\
\hline \multicolumn{2}{|l|}{$60-69$} & +1 \\
\hline \multicolumn{2}{|l|}{$\geq 70$} & 0 \\
\hline \multicolumn{3}{|c|}{ SCORE INTERPRETATION } \\
\hline $\begin{array}{l}\text { RoPE } \\
\text { score }\end{array}$ & $\begin{array}{c}\text { PFO-attributable } \\
\text { fraction } \\
(95 \% \mathrm{CI})\end{array}$ & $\begin{array}{c}\text { Estimated stroke/ } \\
\text { /TIA recurrence } \\
\text { at } 2 \text { years }\end{array}$ \\
\hline $0-3$ & $0 \%(0-4)$ & $20 \%(12-28)$ \\
\hline 4 & $38 \%(25-48)$ & $12 \%(6-18)$ \\
\hline 5 & $34 \%(21-45)$ & $7 \%(3-11)$ \\
\hline 6 & $62 \%(54-68)$ & $8 \%(4-12)$ \\
\hline 7 & $72 \%(66-76)$ & $6 \%(2-10)$ \\
\hline 8 & $84 \%(79-87)$ & $6 \%(2-10)$ \\
\hline $9-10$ & $88 \%(83-91)$ & $2 \%(0-4)$ \\
\hline
\end{tabular}

$\mathrm{Cl}$ - confidence interval; TIA - transient ischemic attack

a potential source of embolism. Some data suggest that when a foramen ovale closes spontaneously, the septum primum and the septum secundum fuse initially at the caudal limit of the zone of overlap of the two structures (Fig. 2). This incomplete fusion results in a pouch that, in the majority of instances, communicates with the left atrium cavity [40]. The presence of LASP with access to the systemic circulation also raises the possibility that, similar to the LAA, during low-flow states, this pouch might serve as a site for thrombus formation and embolization. But the results of clinical studies are inconclusive [30].

In a case-control study of relatively older subjects (mean age 69 years), Tugcu et al. [33] found that LASP was not associated with ischemic or cryptogenic strokes. Negative results between LASP and cryptogenic stroke were also reported in a cohort study of 566 consecutive patients undergoing TEE by Wayangankar et al. [34]. The same author also carried out a retrospective casecontrol study in patients with a high prevalence of cardiovascular risk factors. The prevalence of LASP was $18 \%$, irrespective of age or pathology. Their study did not show any association between LASP and ischemic stroke or cryptogenic stroke [35]. On the other hand, Wong et al. [35] studied 212 consecutive TEE patients with mean age 57 years and found an increased prevalence of LASP among cryptogenic stroke patients compared to ischemic stroke patients of other subtypes. Sun et al. [31] demonstrated among 324 patients, evidence of an association between LASP and ischemic stroke in either univariable analysis and after adjustment for other stroke risk factors using multiple logistic regression analysis. The risk of ischemic stroke in this study was two- fold among patients with LASP than cases without LASP. Additional investigation should take place to determine the clinical significance of LASP and what interventions are required to prevent ischemic stroke in at-risk individuals [31].

\section{The Eustachian valve}

The EV is a remnant of the embryonic right valve of the sinus venosus. Embryologically, the EV directs oxygenated blood from the inferior vena cava across the PFO into the systemic circulation. By directing the blood from the inferior cava to the interatrial septum, a persisting EV may prevent spontaneous closure of PFO after birth and may, therefore, indirectly predispose one to paradoxical embolism. However, there have been relatively few studies performed looking at stroke risk in this population and not all authors agree that prominent $\mathrm{EV}$ predisposes to additional risk. In a study by Schuchlenz et al. [36] the mean EV size was $1.0 \pm 0.4 \mathrm{~cm} ; 70 \%$ of patients with an EV had a PFO $(\mathrm{p}<0.001)$. In contrast the prevalence of PFO in the control group was $30 \%$ and $61 \%$ for those with presumed paradoxical embolism $(\mathrm{p}<0.001)$. Thus, an EV was significantly more common for patients with presumed paradoxical embolism than in control patients ( 143 of $211-68 \%$ vs. 31 of 95 $-33 \%$, respectively, $\mathrm{p}<0.001$ ) [36].

The Vale et al. [37] investigated the relationship between EV length and atrial septal movement in 72 consecutive patients referred to their centre for PFO closure following presumed cryptogenic stroke. The authors proposed that while a large degree of atrial septal movement significantly increases propensity to cerebral embolism in patients with PFO, its absence does not negate this risk. They have shown that long $\mathrm{EV}$ may function 


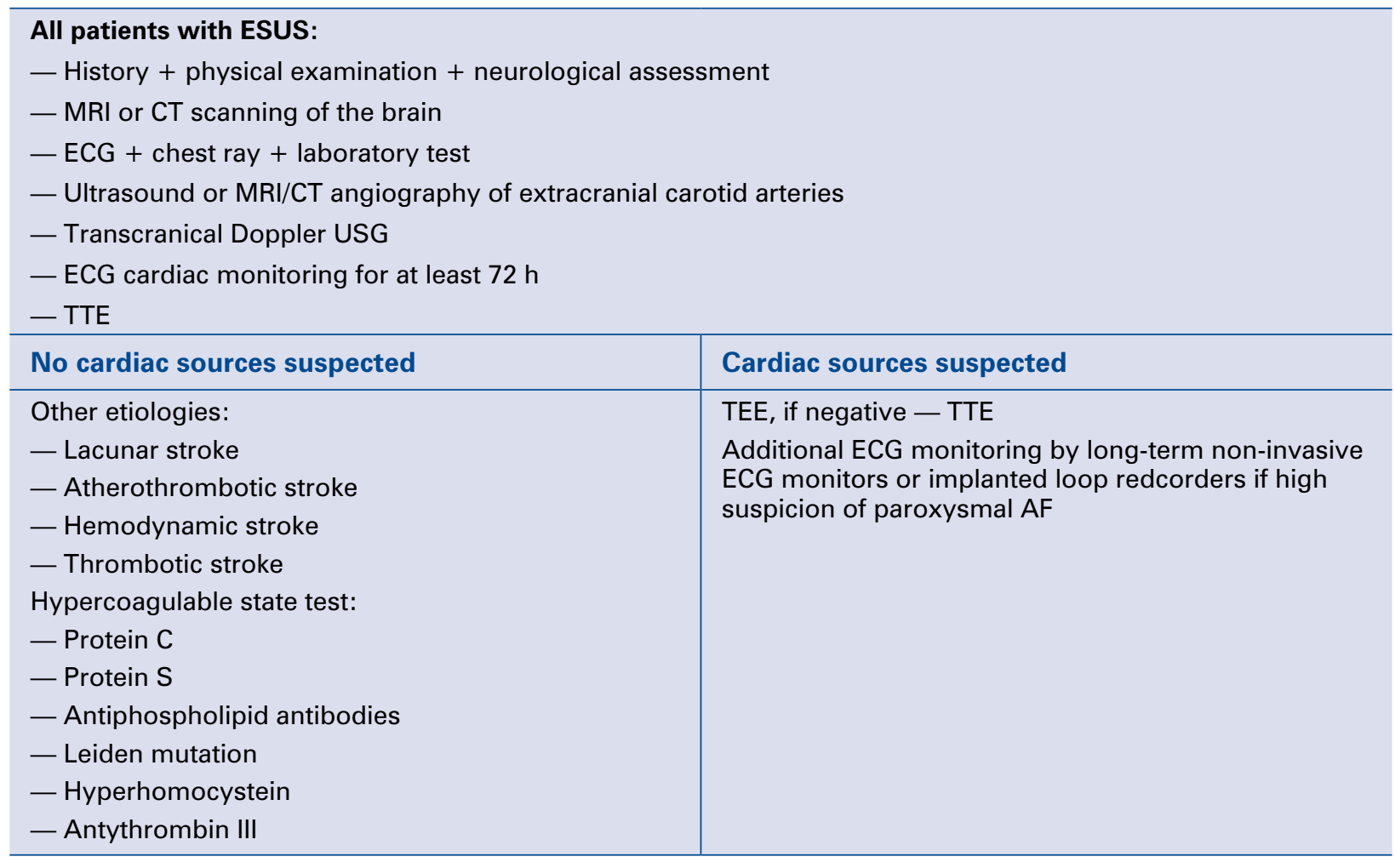

Figure 2. Diagnostic algorithm for cardiac workup of ischemic stroke. AF — atrial fibrillation; CT - computed tomography; ECG - electrocardiography; ESUS - stroke of undetermined source; MRI - magnetic resonance imaging; USG - ultrasonography; TEE — transesophageal echocardiography; TTE — transthoracic echocardiography.

independently from atrial septal movement to potentiate paradoxical embolism [37].

\section{Chiari network}

The $\mathrm{CN}$, present in approximately $2 \%$ of the population, and is a reticulated network of fibers originating from the Eustachian connecting to different parts of the right atrium. Its presence results from incomplete reabsorption of the right valve of the sinus venosus [41]. $\mathrm{CN}$ is often clinically insignificant. However, it has been reported to be involved in the pathogenesis of thromboembolic disease, endocarditis, arrhythmias, and entrapment of catheters upon percutaneous intervention [42]. The network is associated with PFO in $80 \%$ of cases. The role of a $\mathrm{CN}$ in the pathogenesis of stroke is discussed. $\mathrm{CN}$ may create turbulent blood flow leading to thrombus formation. The fibers of the network are sometimes torn during life and may break free. The fenestrated types may rarely remove emboli from the circulation, but this is purely by chance, and further emboli are likely to reach the lung [38]. Rigatelli et al. [39] during over a 24 -month period, prospectively enrolled 50 consecutive patients (mean age $37 \pm 12.5$ years,
38 females) with previous stroke and migraine referred for $\mathrm{PFO}$ catheter-based closure. Patients with $\mathrm{EV}$ and $\mathrm{CN}$ had more frequently a curtain pattern on transcranical Doppler, a larger rightto-left shunt, more recurrent cerebral paradoxical embolism before closure, and a higher preoperative MIDAS score. This study suggests that EV and $\mathrm{CN}$ have a deep impact on migraine with aura and paradoxical embolism pathophysiology: $\mathrm{EV}, \mathrm{CN}$, and migraine with aura should be considered as adjunctive risk factors for paradoxical embolism in the work-up of both symptomatic and asymptomatic PFO patients [39].

\section{The left atrial enlargement}

Little is known about the risk of stroke associated with left atrial enlargement in patients in sinus rhythm, and whether such patients may need a thromboprophylaxis. Formal stroke risk stratification among patients with left atrial enlargement may further help to identify patients who stand to gain from preventive antithrombotic therapy [43]. The normal left atrial volume index (LAVI) using echocardiography is $22 \pm 6 \mathrm{~mL} / \mathrm{m}^{2}$; thus, on the basis of the sensitivity and specificity 
for predicting cardiac events [3, 6-8], the American Society of Echocardiography (ASE) considers left atrial enlargement as $>28 \mathrm{~mL} / \mathrm{m}^{2}$ (i.e., 1 SD from the mean). However, for the purpose of identifying LV diastolic dysfunction, an LAVI cut point $>34 \mathrm{~mL} / \mathrm{m}^{2}$ (i.e., $2 \mathrm{SD}$ ) was endorsed in both ASE and the European Association of Echocardiography guideline document [26]. Biteker et al. [44] prospectively followed 310 consecutive first-ever acute ischemic stroke patients aged 50 years or older who were admitted to hospital within $24 \mathrm{~h}$ of the onset of stroke symptoms. The optimal cut off value, sensitivity, and specificity of LAVI to distinguish cardioembolic stroke from non-cardioembolic stroke were $30 \mathrm{~mL} / \mathrm{m}^{2}$ ( $81 \%$ and $64 \%$, respectively). Kaplan-Meier analysis showed that there was a stepwise increase in risk of mortality with each increment of LAVI category. Overvad et al. [40] identified nine cohort studies and analyzed a total of 67,875 participants and 3,093 stroke outcomes. All studies reported a higher risk of stroke with larger/enlarged left atrium compared to smaller/normal sized left atrium. The underlying etiology explaining this observed higher risk is likely to be multifactorial and not confined to a potential direct effect of left atrial enlargement on thromboembolic risk [44]. In the study of Yaghi et al. [41] in 655 first ischemic stroke patients over a median of 4 years, there were 65 recurrent ischemic strokes (29 were cardioembolic or cryptogenic). In multivariable models adjusted for confounders, including AF and heart failure, moderate-severe left atrial enlargement compared with normal left atrial size was associated with greater risk of recurrent cardioembolic/cryptogenic stroke, but not total ischemic stroke [42].

Left atrial dysfunction has been reported in patients with PFO. It is postulated that left atrial dysfunction could be involved in the development of an arterial embolism in patients with PFO [43]. One hypothesis is that a pathological PFO might contribute to atrial enlargement. In contrast, another possibility is that cardiac thrombus that is secondary to left atrial enlargement might be a determinant of stroke and PFO is additive or even incidental.

\section{Atrial septal aneurysm}

An ASA consists of redundant atrial septal tissue bulging into the right or the left atrium (Fig. 1). Some studies indicate that existence of ASA may be a potential risk factor of stroke. In patients with ASA and a history of embolic events, ASA may enhance migration of a thrombus con- stituted in situ or transiting through it. Marked mobility of ASA may also increase the risk of peripheral embolus. Rigatelli et al. [39] revealed that moderate-to-severe ASA might be associated with left atrial dysfunction in patients with PFO. In the majority of cases ASA is associated with other cardiac abnormalities such as PFO and atrial septal defects as well as mitral valve prolapse or atrial arrhythmias. Mattioli et al. [22] have demonstrated a statistical association between PFO and ASA and have shown that both morphological abnormalities were independent predictors of embolic events in a multivariate analysis [44].

\section{Anticoagulation affairs and stroke risk}

Acetylsalicylic acid, clopidogrel, or the combination of acetylsalicylic acid and dipyridamole, are all acceptable options for secondary prevention in patients with ischemic stroke or TIA of arterial origin [45]. Currently, OAC therapy with warfarin or one of the non-vitamin $\mathrm{K}$ antagonist oral anticoagulants (NOACs), including a direct thrombin inhibitor (dabigatran) and factor Xa inhibitors (rivaroxaban, apixaban, and edoxaban) is the most effective prophylactic approach available to patients with $\mathrm{AF}$ at high risk of thromboembolic events. Contemporary guidelines from the European Society of Cardiology, the American College of Cardiology, the American Heart Association (AHA), and the Heart Rhythm Society for managing stroke prophylactic treatment in $\mathrm{AF}$ patients advocate for the use of the $\mathrm{CHA}_{2} \mathrm{DS}_{2}$-VASc score (Congestive heart failure [1 point], Hypertension [1 point], Age 75 years [2 points], Diabetes [1 point], Stroke [2 points], Vascular disease [1 point], Age $65-74$ years [1 point], and female sex category [1 point]) for stroke risk stratification [46, 47]. In 'low risk' patients with no additional stroke risk factors, oral anticoagulant treatment is not recommended. The United States guideline offers a Class IIb recommendation on either no treatment, acetylsalicylic acid therapy, or oral anticoagulant treatment in $\mathrm{AF}$ patients with a $\mathrm{CHA}_{2} \mathrm{DS}_{2}$-VASc score of 1 . The European guideline recommends that female sex as a single risk factor should be disregarded, and hence, low risk is defined as a $\mathrm{CHA}_{2} \mathrm{DS}_{2}$-VASc score of 0 in males, 1 in females, where no antithrombotic therapy is recommended [48, 49]. Thus, oral anticoagulant treatment should be considered in $\mathrm{AF}$ patients with a one stroke risk factor (i.e. $\mathrm{CHA}_{2} \mathrm{DS}_{2}-$ -VASc score of 1 in males, or 2 for females; Class IIa recommendation), or recommended in those with a $\mathrm{CHA}_{2} \mathrm{DS}_{2}$-VASc score $\geq 2$ (Class I recommendation) $[50,51]$. Patients with mechanical valve prosthesis 
and with AF and moderate or severe MS should be treated with vitamin $\mathrm{K}$ antagonist. There is a high risk of stroke and systemic thromboembolism but also a high risk of bleeding if anticoagulants are prescribed. Newer surgical or percutaneous interventions (WATCHMAN, LARIAT, and Amplatzer devices) have been developed for stroke prevention in patients with AF. These devices are typically used in $\mathrm{AF}$ patients who have high risk for thromboembolic events and cannot tolerate prolonged anticoagulation therapy. Although these interventions have shown feasibility, their longterm superiority to medical management remains a matter of debate [12]. The cryptogenic strokes are being recognized as sharing many characteristics with cardioembolic strokes [4]. The major challenge in supervising stroke is secondary stroke prevention of cryptogenic strokes, especially in choosing antithrombotic therapy [4]. A relatively frequent complication of ischemic stroke is hemorrhagic transformation. It happens in $2.2 \%$ to $44 \%$ of clinical cases [51]. There has been no definitive study assessing the comparative effectiveness of $\mathrm{OAC}$ vs. APT in this population. Currently available data do not provide definitive evidence on the comparative benefits of OAC vs. APT in patients with cryptogenic stroke or ESUS [44]. There are ongoing studies assessing advantages and disadvantages of these two kinds of therapies. The Randomized, double-blind, Evaluation in secondary Stroke Prevention comparing the EfficaCy and safety of the oral Thrombin inhibitor dabigatran etexilate vs. acetylsalicylic acid in patients with Embolic Stroke of Undetermined Source (RE-SPECT ESUS) is a prospective, randomized, double-blind, multicenter trial involving approximately 6000 patients and 550 centers. Subjects are randomized to dabigatran or acetylsalicylic acid and are treated for an expected minimum of 6-months and for up to approximately 3 years. The primary efficacy outcome is a time to first recurrent stroke (ischemic, hemorrhagic, or unspecified). Key secondary outcomes are time to first ischemic stroke and time to first occurrence in the composite outcome of nonfatal stroke, nonfatal MI, and cardiovascular death. The primary safety outcome is major hemorrhage, including symptomatic intracranial hemorrhage [50]. The ATTICUS randomized trial is designed to determine whether the factor Xa inhibitor apixaban administered within 7 days after ESUS, is superior to acetylsalicylic acid for prevention of new ischemic lesions documented by brain MRI within 12 months after index stroke. The primary outcome is the occurrence of at least one new ischemic le- sion identified by axial T2-weighted FLAIR MRI and/or axial diffusion-weighted imaging MRI at 12 months when compared with the baseline MRI. Key secondary outcomes are the combination of recurrent ischemic strokes, hemorrhagic strokes, and systemic embolism [52].

The New Approach riVaroxaban Inhibition of Factor Xa in a Global trial versus ASA to prevent Embolism in ESUS (NAVIGATE ESUS) is a multinational, randomized, double-blind, superiority trial comparing antithrombotic therapies for secondary stroke prevention in a well-defined cohort of patients with nonlacunar cryptogenic stroke with embolic features. Main results are anticipated in 2018.

There is a possibility that a covert AF is the underlying pathogenesis in $\approx 40 \%$ of ESUS patients. In this context the present antithrombotic strategy might be suboptimal, which in turn could have important consequences on their outcome. Indeed, the reduced risk of ischemic stroke in response to warfarin is offset by a risk of hemorrhage in patients with reduced LVEF [51]. Therefore, it is not clear whether patients with sinus rhythm and reduced LV function should be treated with anticoagulation therapy during or after treatment for heart failure [53]. The ACCF/AHA guidelines for the management of valvular heart disease recommend acetylsalicylic acid therapy for patients with mitral valve prolapse who experience TIAs (Class I; Level of evidence C) and warfarin for these patients with a history of stroke and mitral regurgitation, $\mathrm{AF}$, or left atrial thrombus (Class I; Level of evidence C). There is no evidence that anticoagulant therapy reduces the risk of stroke in patients with mitral annular calcification (MAC) [21]. The decision to use antiplatelet agents vs. anticoagulants in patients with MAC should include the consideration of other potential comorbid factors such as: AF (that can occur 12 times more often in patients with MAC in comparison to those without MAC) or endocarditics [26,53]. Current evidence-based guidelines do not support routine closure of PFO in the absence of deep venous thrombus or proximal source and recommend antiplatelet therapy. Three major randomized controlled trials did not show a net benefit from closure of $\mathrm{PFO}$ in this population. A meta-analysis evaluating these 3 and an additional 11 non-randomized observational studies failed to prove superiority of closure against medical therapy, with an increased incidence of new onset AF in the closure group (RR 3.50). However, it should be noted that previous PFO closure trials differed in study criteria, devices used, and lack of 
Table 4. Potential sources of embolism and type of anti-thrombotic therapy in patients after ischemic stroke.

\begin{tabular}{|c|c|c|}
\hline \multirow[t]{2}{*}{ Potential source of embolism } & \multicolumn{2}{|c|}{ Type of anti-thrombotic therapy } \\
\hline & Established & Not established \\
\hline \multicolumn{3}{|l|}{ Atrial arrhythmias: } \\
\hline — Valvular AF & $x$ & \\
\hline - Non-valvular AF & $x$ & \\
\hline — Atrial flutter & $\begin{array}{l}\mathrm{x} \\
\text { (anticoagulation according } \\
\text { CHADSVASC score) }\end{array}$ & \\
\hline Prosthetic valves and devices & $\begin{array}{l}\mathrm{x} \\
\text { (prostetic valve }- \text { VKA, device } \\
\text { according indications) }\end{array}$ & \\
\hline Sinus rhythm and reduced LV function & & $x$ \\
\hline $\begin{array}{l}\text { Patients with mitral valve prolapse who } \\
\text { experience TIAs }\end{array}$ & x (antiplatelet therapy) & \\
\hline $\begin{array}{l}\text { Patients with mitral valve prolapsed and } \\
\text { a history of stroke and mitral regurgitation, } \\
A F \text {, or left atrial thrombus }\end{array}$ & x (anticoagulation) & \\
\hline Mitral annular calcification & & $x$ \\
\hline PFO/ASD without DVT & x (antiplatelet therapy) & \\
\hline PFO/ASD with DVT & x (anticoagulation) & \\
\hline $\begin{array}{l}\text { ASA, Chiari Network, Eustachian valve, } \\
\text { LAE, LASP }\end{array}$ & & $x$ \\
\hline
\end{tabular}

$\mathrm{AF}$ - atrial fibrillation; ASA - atrial septal aneurysm; ASD — atrial septal defect; DVT — deep venous thrombus; LAE — left atrial enlargement; LASP — left atrial septal pouch; LV — left ventricular; PFO — patent foramen ovale; TIA — transient ischemic attack; VKA - vitamin $\mathrm{K}$ antagonist

standardized design. Ongoing trials will hopefully find which patients are ideal for PFO closure. At the present time, patients should be thoroughly evaluated and risk stratified before considering closure of PFO in ESUS [52]. Furthermore, current evidence-based guidelines do not recommend anticoagulation over APT in patients with PFO. A large randomized control trial evaluating outcomes in PFO patients with anticoagulation vs. APT with acetylsalicylic acid reported a 2-year event rates of $9.5 \%$ in the warfarin-treated group and $17.9 \%$ in the acetylsalicylic acid-treated group, but could not conclude statistical significance (HR 0.5; 95\% CI $0.2-1.7)$. In addition, there was no significant difference between patients with isolated PFO, those associated with an atrial septal defect, or among small or large PFO's. More recently, an individual participant meta-analysis evaluating OAC or APT in 2385 patients found no statistically significant difference in recurrent stroke, TIA, death; or stroke alone. Furthermore, subgroup analysis did not find significant heterogeneity of treatment effects in both groups, supporting the finding $[8,53]$. Patients with cryptogenic strokes should be evaluated for the presence of venous thromboembolism. If ve- nous thromboembolism is present, treatment is the same as for pulmonary embolism: anticoagulation. If venous thromboembolism is not present, APT is indicated [54].

\section{Conclusions}

About $80 \%$ of ischemic strokes occur in persons without AF, and it is therefore important to develop a path to examine the optimal prevention of stroke when there is no obvious AF. The benefits of oral anticoagulation for patients with heart failure in sinus rhythm have yet to be established. Many cryptogenic strokes/ESUS are presumed to have an embolic etiology. There are ongoing studies: Re-SPECT ESUS, ATTICUS and NAVIGATE ESUS assessing the comparative effectiveness of OAC vs. APT in patients after ESUS. Further research is needed to determine whether anticoagulant use may reduce risk of recurrence in ischemic stroke patients with moderate to severe left atrial enlargement and other potential sources of thromboembolism (Table 4). At present there is a lack of antithrombothic treatment scheme in the time between stroke 
and finishing the diagnosis of potential sources of thromboembolism in ESUS. It should be discussed if OAC therapy should not be introduced for about 3 months during which patient could have at least 7 days electrocardiogram monitoring, $\mathrm{PFO}$ and deep veins assessment, eventually genetic assessment and based on these results a final decision on treatment can be stated.

\section{Acknowledgements}

The study was financed by research grant no. 502-03/5-139-02/502-54-203 of the Medical University of Lodz.

\section{Conflict of interest: None declared}

\section{References}

1. Li X, Zhou G, Zhou X, et al. The efficacy and safety of aspirin plus dipyridamole versus aspirin in secondary prevention following TIA or stroke: a meta-analysis of randomized controlled trials. J Neurol Sci. 2013; 332(1-2): 92-96, doi: 10.1016/j.jns.2013.06.025, indexed in Pubmed: 23871093.

2. Sacco RL, Kasner SE, Broderick JP, et al. American Heart Association Stroke Council, Council on Cardiovascular Surgery and Anesthesia, Council on Cardiovascular Radiology and Intervention, Council on Cardiovascular and Stroke Nursing, Council on Epidemiology and Prevention, Council on Peripheral Vascular Disease, Council on Nutrition, Physical Activity and Metabolism. An updated definition of stroke for the 21st century: a statement for healthcare professionals from the American Heart Association/American Stroke Association. Stroke. 2013; 44(7): 2064-2089, doi: 10.1161/STR.0b013e318296aeca, indexed in Pubmed: 23652265.

3. Chauhan G, Debette S. Genetic Risk Factors for Ischemic and Hemorrhagic Stroke. Curr Cardiol Reports. 2016; 18(12), doi: 10.1007/s11886-016-0804-z.

4. Zhang C, Kasner S. Diagnosis, prognosis, and management of cryptogenic stroke. F1000Res. 2016; 5: 168, doi: 10.12688/ f1000research.7384.1, indexed in Pubmed: 26918178.

5. Pepi M, Evangelista A, Nihoyannopoulos P, et al. European Association of Echocardiography. Recommendations for echocardiography use in the diagnosis and management of cardiac sources of embolism: European Association of Echocardiography (EAE) (a registered branch of the ESC). Eur J Echocardiogr. 2010; 11(6): 461-476, doi: 10.1093/ejechocard/jeq045, indexed in Pubmed: 20702884.

6. Minematsu K, Yamaguchi T, Omae T. ,Spectacular shrinking deficit': rapid recovery from a major hemispheric syndrome by migration of an embolus. Neurology. 1992; 42(1): 157-162, indexed in Pubmed: 1734297.

7. Turagam MK, Velagapudi P, Flaker GC. Stroke prevention in the elderly atrial fibrillation patient with comorbid conditions: focus on non-vitamin $\mathrm{K}$ antagonist oral anticoagulants. Clin Interv Aging. 2015; 10: 1431-1444, doi: 10.2147/CIA.S80641, indexed in Pubmed: 26366064.

8. Hart RG, Diener HC, Coutts SB, et al. Cryptogenic Stroke/ESUS International Working Group. Embolic strokes of undetermined source: the case for a new clinical construct. Lancet Neurol.
2014; 13(4): 429-438, doi: 10.1016/S1474-4422(13)70310-7, indexed in Pubmed: 24646875.

9. Papavasileiou V, Milionis H, Michel P, et al. ASTRAL score predicts 5-year dependence and mortality in acute ischemic stroke. Stroke. 2013; 44(6): 1616-1620, doi: 10.1161/STROKEAHA.113.001047, indexed in Pubmed: 23559264.

10. Leithäuser B, Park JW. Cardioembolic stroke in atrial fibrillation-rationale for preventive closure of the left atrial appendage. Korean Circ J. 2009; 39(11): 443-458, doi: 10.4070/ /kcj.2009.39.11.443, indexed in Pubmed: 19997539.

11. Nakanishi K, Homma S. Role of echocardiography in patients with stroke. J Cardiol. 2016; 68(2): 91-99, doi: 10.1016/j. jjcc.2016.05.001, indexed in Pubmed: 27256218.

12. Christiansen CB, Gerds TA, Olesen JB, et al. Atrial fibrillation and risk of stroke: a nationwide cohort study. Europace. 2016; 18(11): 1689-1697, doi: 10.1093/europace/euv401, indexed in Pubmed: 26838693.

13. Dalen JE, Alpert JS. Silent atrial fibrillation and cryptogenic strokes. Am J Med. 2017; 130(3): 264-267, doi: 10.1016/j.amjmed.2016.09.027, indexed in Pubmed: 27756556.

14. Olsen FJ, Pedersen S, Jensen JS, et al. Global longitudinal strain predicts incident atrial fibrillation and stroke occurrence after acute myocardial infarction. Medicine (Baltimore). 2016; 95(44): e5338, doi: 10.1097/MD.0000000000005338, indexed in Pubmed: 27858918.

15. Lee AY. Cancer and thromboembolic disease: pathogenic mechanisms. Cancer Treat Rev. 2002; 28(3): 137-140, indexed in Pubmed: 12234564 .

16. Stöllberger C, Wegner C, Finsterer J. CHADS2- and CHA2DS2VASc scores and embolic risk in left ventricular hypertrabeculation/noncompaction. J Stroke Cerebrovasc Dis. 2013; 22(6): 709712, doi: $10.1016 /$ j.jstrokecerebrovasdis.2011.10.014, indexed in Pubmed: 22142779.

17. Wigger O, Windecker S, Bloechlinger S. Nonbacterial thrombotic endocarditis presenting as intracerebral hemorrhage. Wien Klin Wochenschr. 2016; 128(23-24): 922-924, doi: 10.1007/s00508016-1020-y, indexed in Pubmed: 27325214.

18. Gundersen H, Moynihan B. An Uncommon Cause of Stroke: Non-bacterial Thrombotic Endocarditis. Journal of Stroke and Cerebrovascular Diseases. 2016; 25(10): e163-e164, doi: 10.1016/j.jstrokecerebrovasdis.2015.11.009.

19. Bjessmo S, Ivert T. Cardiac myxoma: 40 years' experience in 63 patients. Ann Thorac Surg. 1997; 63(3): 697-700, indexed in Pubmed: 9066386.

20. Akdemir I, Dagdelen S, Yuce M, et al. Silent brain infarction in patients with rheumatic mitral stenosis. Jpn Heart J. 2002; 43(2): 137-144, indexed in Pubmed: 12025900.

21. González A, Altieri P, Màrquez E, et al. Massive pulmonary embolism associated with a right ventricular myxoma. Am J Med. 1980; 69(5): 795-798, doi: 10.1016/0002-9343(80)90455-6.

22. Mattioli AV, Aquilina M, Oldani A, et al. Atrial septal aneurysm as a cardioembolic source in adult patients with stroke and normal carotid arteries. A multicentre study. Eur Heart J. 2001; 22(3): 261-268, doi: 10.1053/euhj.2001.2293, indexed in Pubmed: 11161938.

23. Ihsen Z, Hela M, Khadija M, et al. Cerebral embolism complicating left atrial myxoma: a case report. Pan Afr Med J. 2016; 24: 140, doi: 10.11604/pamj.2016.24.140.9778, indexed in Pubmed: 27642478 .

24. Merkler AE, Chu SY, Lerario MP, et al. Temporal relationship between infective endocarditis and stroke. Neurology. 2015; 
85(6): 512-516, doi: 10.1212/WNL.0000000000001835, indexed in Pubmed: 26163428.

25. Zhang F, Zhu Z, Upadhya GK, et al. Papillary fibroelastoma of the aortic valve presenting with chronic angina and acute stroke: a case report. J Med Case Rep. 2017; 11(1): 17, doi: 10.1186/ s13256-016-1179-x, indexed in Pubmed: 28095921.

26. Krishnan SC, Salazar M. Septal pouch in the left atrium: a new anatomical entity with potential for embolic complications. JACC Cardiovasc Interv. 2010; 3(1): 98-104, doi: 10.1016/j. jcin.2009.07.017, indexed in Pubmed: 20129577.

27. Li L, Yiin GS, Geraghty OC, et al. Oxford Vascular Study. Incidence, outcome, risk factors, and long-term prognosis of cryptogenic transient ischaemic attack and ischaemic stroke: a population-based study. Lancet Neurol. 2015; 14(9): 903913, doi: 10.1016/S1474-4422(15)00132-5, indexed in Pubmed: 26227434.

28. Akhtar J, Wasay M, Rauf J. Atrial myxoma: a rare cause of cardioembolic stroke. BMJ Case Rep. 2012; 2012, doi: 10.1136/ bcr.2012.006176, indexed in Pubmed: 22962389.

29. Kraywinkel K, Jauss M, Diener HC, et al. [Patent foramen ovale, atrial septum aneurysm, and stroke. An examination of the status of recent evidence]. Nervenarzt. 2005; 76(8): 935-942, doi: 10.1007/s00115-004-1874-5, indexed in Pubmed: 15696307.

30. Rao PA, Nagendra Prakash SN, Vasudev S, et al. A rare case of right ventricular myxoma causing recurrent stroke. Indian Heart J. 2016; 68 Suppl 2: S97-S101, doi: 10.1016/j.ihj.2016.05.001, indexed in Pubmed: 27751344.

31. Sun JP, Meng F, Yang XS, et al. Prevalence of atrial septal pouch and risk of ischemic stroke. Int J Cardiol. 2016; 214: 37-40, doi: 10.1016/j.ijcard.2016.03.119, indexed in Pubmed: 27057971.

32. Homma S, Messé SR, Rundek T, et al. Patent foramen ovale. Nat Rev Dis Primers. 2016; 2: 15086, doi: 10.1038/nrdp.2015.86, indexed in Pubmed: 27188965.

33. Tugcu A, Okajima K, Jin Z, et al. Septal pouch in the left atrium and risk of ischemic stroke. JACC Cardiovasc Imaging. 2010; 3(12): 1276-1283, doi: 10.1016/j.jcmg.2010.11.001, indexed in Pubmed: 21163457.

34. Wayangankar SA, Patel JH, Patel B, et al. Clinical and echocardiographic variables associated with LA septal pouch. JACC Cardiovasc Imaging. 2013; 6(7): 833-835, doi: 10.1016/j. jcmg.2012.09.021, indexed in Pubmed: 23664719.

35. Wong JM, Lombardo DM, Barseghian A, et al. Left atrial septal pouch in cryptogenic stroke. Front Neurol. 2015; 6: 57, doi: 10.3389/fneur.2015.00057, indexed in Pubmed: 25852636.

36. Schuchlenz HW, Saurer G, Weihs W, et al. Persisting eustachian valve in adults: relation to patent foramen ovale and cerebrovascular events. J Am Soc Echocardiogr. 2004; 17(3): 231-233, doi: 10.1016/j.echo.2003.12.003, indexed in Pubmed: 14981420.

37. Vale TA, Newton JD, Orchard E, et al. Prominence of the Eustachian valve in paradoxical embolism. Eur J Echocardiogr. 2011; 12(1): 33-36, doi: 10.1093/ejechocard/jeq100, indexed in Pubmed: 20813791.

38. Jansirani DD, Deep SS, Anandaraja S. Anatomical study of Chiari network and the remnant of left venous valve in the interior of right atrium. Anat Res Int. 2015; 2015: 247680, doi: 10.1155/2015/247680, indexed in Pubmed: 26442159 .

39. Rigatelli G, Dell'avvocata F, Cardaioli P, et al. Migraine-patent foramen ovale connection: role of prominent eustachian valve and large Chiari network in migrainous patients. Am J Med Sci. 2008; 336(6): 458-461, doi: 10.1097/MAJ.0b013e31816e189d, indexed in Pubmed: 19092317.

40. Overvad TF, Nielsen PB, Larsen TB, et al. Left atrial size and risk of stroke in patients in sinus rhythm. A systematic review.
Thromb Haemost. 2016; 116(2): 206-219, doi: 10.1160/TH15-120923, indexed in Pubmed: 27075168.

41. Yaghi S, Chang AD, Hung P, et al. Left Atrial Appendage Morphology and Embolic Stroke of Undetermined Source: A Cross-Sectional Multicenter Pilot Study. J Stroke Cerebrovasc Dis. 2018; 27(6): 1497-1501, doi: 10.1016/j.jstrokecerebrovasdis.2017.12.036, indexed in Pubmed: 29398537.

42. Nouh A, Hussain M, Mehta T, et al. Embolic strokes of unknown source and cryptogenic stroke: implications in clinical practice. Front Neurol. 2016; 7: 37, doi: 10.3389/fneur.2016.00037, indexed in Pubmed: 27047443.

43. Homma S, Sacco RL, Di Tullio MR, et al. PFO in Cryptogenic Stroke Study (PICSS) Investigators. Effect of medical treatment in stroke patients with patent foramen ovale: patent foramen ovale in Cryptogenic Stroke Study. Circulation. 2002; 105(22): 2625-2631, indexed in Pubmed: 12045168.

44. Biteker M, Kayataş K, Başaran Ö, et al. The role of left atrial volume index in patients with a first-ever acute ischemic stroke. J Stroke Cerebrovasc Dis. 2017; 26(2): 321-326, doi: 10.1016/j.jstrokecerebrovasdis.2016.09.023, indexed in Pubmed: 27773589.

45. Rigatelli G, Aggio S, Cardaioli P, et al. Left atrial dysfunction in patients with patent foramen ovale and atrial septal aneurysm: an alternative concurrent mechanism for arterial embolism? JACC Cardiovasc Interv. 2009; 2(7): 655-662, doi: 10.1016/j. jcin.2009.05.010, indexed in Pubmed: 19628189.

46. Hoit BD. Left atrial size and function: role in prognosis. J Am Coll Cardiol. 2014; 63(6): 493-505, doi: 10.1016/j.jacc.2013.10.055, indexed in Pubmed: 24291276.

47. Mehrpour M, Mehrpour M. Is the serum ferritin level a considerable predictor for hemorrhagic transformation of ischemic stroke? Med J Islam Repub Iran. 2016; 30: 363, indexed in Pubmed: 27493907.

48. Meier B, Kalesan B, Mattle HP, et al. PC Trial Investigators. Percutaneous closure of patent foramen ovale in cryptogenic embolism. N Engl J Med. 2013; 368(12): 1083-1091, doi: 10.1056/ NEJMoa1211716, indexed in Pubmed: 23514285.

49. Wolfrum M, Froehlich GM, Knapp G, et al. Stroke prevention by percutaneous closure of patent foramen ovale: a systematic review and meta-analysis. Heart. 2014; 100(5): 389-395, doi: 10.1136/heartjnl-2013-304394, indexed in Pubmed: 23793373.

50. Dalen JE, Alpert JS. Cryptogenic strokes and patent foramen ovales: what's the right treatment? Am J Med. 2016; 129(11): 1159-1162, doi: 10.1016/j.amjmed.2016.08.006, indexed in Pubmed: 27566504.

51. Wong JM, Fisher M. The potential role of the left atrial septal pouch in cryptogenic stroke. Expert Rev Cardiovasc Ther. 2016; 14(1): 1-3, doi: 10.1586/14779072.2015.1100536, indexed in Pubmed: 26488338.

52. Hart RG, Sharma M, Mundl H, et al. Rivaroxaban for secondary stroke prevention in patients with embolic strokes of undetermined source: Design of the NAVIGATE ESUS randomized trial. Eur Stroke J. 2016; 1(3): 146-154.

53. Geisler T, Poli S, Meisner C, et al. Apixaban for treatment of embolic stroke of undetermined source (ATTICUS randomized trial): Rationale and study design. Int J Stroke. 2017; 12(9): 985-990, doi: 10.1177/1747493016681019, indexed in Pubmed: 27881833.

54. Glass TA, Hennessey PM, Pazdera L, et al. Outcome at 30 days in the New England Medical Center Posterior Circulation Registry. Arch Neurol. 2002; 59(3): 369-376, indexed in Pubmed: 11890839. 